\title{
Rare decays at CMS
}

\author{
Kai-Feng Chen* \\ National Taiwan University \\ E-mail: kf jack@phys.ntu.edu.tw \\ on behalf of the CMS Collaboration
}

With the large integrated luminosity collected and the high efficiency of its di-muon triggers, the CMS experiment is a privileged test bench for rare decays involving heavy flavours. We report about the most recent measurements based on LHC data, including decays of B particles to muons and rare vector boson decays to heavy flavours.

ICHEP2018, 39th International Conference on High Energy Physics

4-11 July 2018

Seoul, Korea

${ }^{*}$ Speaker. 
Probing for new physics beyond the standard model (SM) is the major goal of the LHC experiments. One can perform direct searches by producing real new particles at high energy collisions and the particles can be discovered via their decays or any other interactions with the detectors. Alternatively indirect searches can be carried out if the new particles virtually participate in the decay processes, and the effects can be observed by seeing any deviations from the SM predictions. Both direct and indirect searches are necessary and complement with each other. The presented analyses are performed using the data collected by the CMS experiment [1] in pp collisions.

\section{Search for rare $B_{s} \rightarrow \mu^{+} \mu^{-}$and $B^{0} \rightarrow \mu^{+} \mu^{-}$decays}

The $B_{s} \rightarrow \mu^{+} \mu^{-}$and $B^{0} \rightarrow \mu^{+} \mu^{-}$decays are expected to be extremely small in the SM since these processes only proceed through flavor-changing neutral currents (FCNC), which are not allowed at the leading order. The internal annihilation of quarks in the $B_{s}$ and $B_{d}$ mesons is needed and the rate is further suppressed. Another suppression factor, the squared of the ratio between the muon and $B$-meson masses, $m_{\mu}^{2} / m_{B}^{2}$, is again required by the helicity. The branching fractions of these two modes in SM are calculated [2] to be as small as $\mathscr{B}^{\mathrm{SM}}\left(B_{s} \rightarrow \mu^{+} \mu^{-}\right)=$ $(3.66 \pm 0.23) \times 10^{-9}$ and $\mathscr{B}^{\mathrm{SM}}\left(B^{0} \rightarrow \mu^{+} \mu^{-}\right)=(1.06 \pm 0.09) \times 10^{-10}$.

Thanks to the large production cross section of $b$-hadrons at the LHC, these two decays become accessible even with SM branching fractions. However several SM extended theories may enlarge the branching fractions by a significant factor. In the MSSM models the branching fraction of $B_{s} \rightarrow$ $\mu^{+} \mu^{-}$has a strong dependence on $\tan \beta$ to the sixth power; by introducing extended Higgs sectors the branching fractions are also enlarged. The ratio of $B^{0} \rightarrow \mu^{+} \mu^{-}$and $B_{s} \rightarrow \mu^{+} \mu^{-}$branching fractions is also a good test of the minimal flavour violation scenarios. By discovering any deviation from the predictions of these two channels could provide a clue how the SM should be extended. On the other hand, if the branching fractions are measured to be consistent with the SM predictions, strong constraints can be adopted to the new physics models.

Due to the small expected number of signal events stacking on thick combinatorial background layer, the main focus of the analysis is how to reduce the background. Signal $B \rightarrow \mu^{+} \mu^{-}$decay is consistent with a pair of real muons and a displaced decay vertex, and their invariant mass should near the known masses of $B_{s}$ and $B^{0}$ mesons. The main source of the background is combinations of muons from the semileptonic decays of $b$-hadrons; hence there can be nearby additional activities other than the candidate muon pair as well as a poor vertexing quality due to the finite $B$ hadron lifetime. All of these information are combined with boosted decision trees (BDTs) to further improve the background suppression capability. The background with mis-identified kaons or pions is also critical: considering a $B$ meson decaying into two hadrons $\left(B \rightarrow K^{+} K^{-}, K^{+} \pi^{-}\right.$, or $\left.\pi^{+} \pi^{-}\right)$ with both hadrons mis-reconstructed as muons, it forms a peak right below the signal $B \rightarrow \mu^{+} \mu^{-}$. In order to suppress such annoying background, a stringent requirement on a BDT-based muon identification is introduced.

The $B_{s} \rightarrow \mu^{+} \mu^{-}$branching fractions are normalized to the $B^{+} \rightarrow J / \psi\left(\rightarrow \mu^{+} \mu^{-}\right) K^{+}$decay, $\mathscr{B}\left(B_{s} \rightarrow \mu^{+} \mu^{-}\right)=\frac{N_{\text {sig }}}{N\left(B^{+} \rightarrow J / \psi K^{+}\right)} \cdot \mathscr{B}\left(B^{+} \rightarrow J / \psi K^{+}\right) \cdot \frac{\varepsilon\left(B^{+}\right)}{\varepsilon\left(B_{s}\right)} \cdot \frac{f_{u}}{f_{s}}$, where $N_{\text {sig }}$ is the signal yield, $\varepsilon$ corrects for the detecting acceptance and efficiency; $f_{u} / f_{s}$ is the ratio of hadronization fractions and is not included for $B^{0} \rightarrow \mu^{+} \mu^{-}$branching fraction. Very similar selection criteria are introduced for the two muons from $J / \psi$ decays, and hence the systematic uncertainties can be mostly cancelled. 
As shown in Figure 1, the $B_{s} \rightarrow \mu^{+} \mu^{-}$channel has been measured using full CMS Run-1 data [3] sets with a significance of 4.3 standard deviations. The resulting branching fraction for $B_{s} \rightarrow \mu^{+} \mu^{-}$and upper limit for $B^{0} \rightarrow \mu^{+} \mu^{-}$are given by $\mathscr{B}\left(B_{s} \rightarrow \mu^{+} \mu^{-}\right)=\left(3.0_{-0.9}^{+1.0}\right) \times 10^{-9}$ and $\mathscr{B}\left(B^{0} \rightarrow \mu^{+} \mu^{-}\right)<1.1 \times 10^{-9}$. The measured branching fraction of $B_{s} \rightarrow \mu^{+} \mu^{-}$is in agreement with the SM prediction.

A combined analysis using CMS and LHCb Run-1 data has been performed [4]. The combined branching fractions are given by $\mathscr{B}\left(B_{s} \rightarrow \mu^{+} \mu^{-}\right)=\left(2.8_{-0.6}^{+0.7}\right) \times 10^{-9}$ and $\mathscr{B}\left(B^{0} \rightarrow \mu^{+} \mu^{-}\right)=$ $\left(3.9_{-1.4}^{+1.6}\right) \times 10^{-10}$. A significance of 6.2 standard deviations observed for $B_{s}$ decay. A mild hint of $B^{0} \rightarrow \mu^{+} \mu^{-}$decay has been seen, but still within the uncertainties from the SM prediction. The ratio of $B^{0}$ and $B_{s}$ branching fractions, which is a very clean test of the SM since the major part of theoretical uncertainties cancelled, has been measured as well.
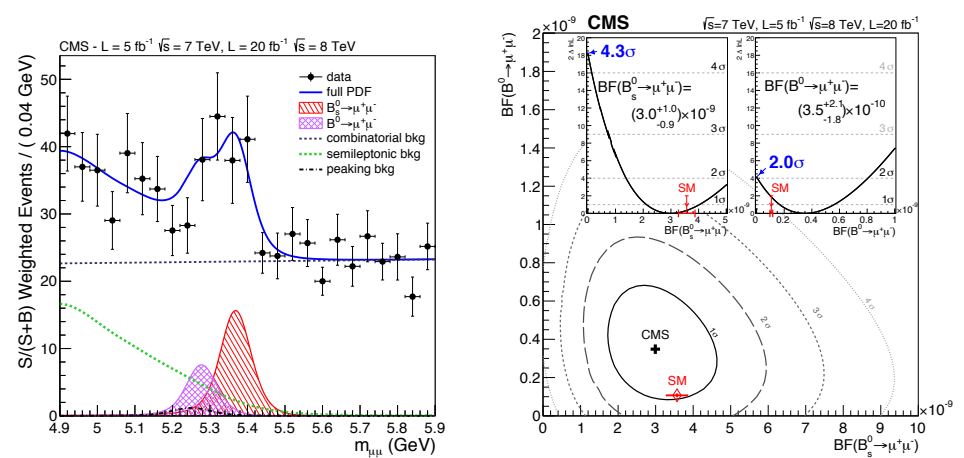

Figure 1: The weighted invariant mass distribution for $B \rightarrow \mu \mu$ candidates (left) and the results of likelihood scans (right) from the CMS Run-1 analysis. Details can be found in Ref. [3].

\section{Search for $Z \rightarrow J / \psi \ell^{+} \ell^{-}$decays}

So far only one exclusive leptonic decay channel $Z \rightarrow 4 \ell$ has been observed apart from the common dilepton final states, although the $Z$ boson has been discovered and very well measured for many years. The search for $Z \rightarrow \gamma \ell \ell$ has been performed but the signal is not yet discovered. In this section the search for $Z$ boson decaying to a $J / \psi$ meson plus a pair of leptons [5] is discussed. The channel has a very clean signature of four leptons final state and it is one of the background for Higgs analysis. Such a decay is sensitive to the fragmentation function for a virtual photon to split into a $J / \psi$, and it has an expected branching fraction in the range of $(6.7-7.7) \times 10^{-7}$. With a larger statistics from upcoming LHC data, possible searches for the signatures from SM-extended models (including anomalous couplings or exotic light states) can be also performed.

The analysis is looking for $Z \rightarrow J / \psi \mu^{+} \mu^{-}$and $J / \psi e^{+} e^{-}$, with subsequent $J / \psi \rightarrow \mu^{+} \mu^{-}$ decay with $35.9 \mathrm{fb}^{-1}$ data collected at $\sqrt{s}=13 \mathrm{TeV}$. The strategy is to first observe the decay itself and to measure the fiducial branching fraction in the detector acceptance. The events are triggered with 1-3 charged leptons with several different $p_{T}$ requirements; using such a mixed trigger scheme a trigger efficiency above $99 \%$ is reached.

Signal yield is obtained from a two-dimentional unbinned extended maximum-likelihood fits to $M(\mu \mu)$ and $M(\mu \mu \ell \ell$ ), with the four components (Z-signal $J / \psi$-signal, $Z$-signal $J / \psi$-background, $Z$-background $J / \psi$-signal, and $Z$-background $J / \psi$-background) included. Clean signal peaks 
are observed in two decay channels: $N\left(Z \rightarrow J / \psi \mu^{+} \mu^{-}\right)=13.0 \pm 3.9$ and $N\left(Z \rightarrow J / \psi e^{+} e^{-}\right)=$ $11.2 \pm 3.4$, where the combined significance of two channels reached $5.7 \sigma$, as a clear discovery of this decay. Figure 2 shows the two-dimentional of $M(\mu \mu)$ and $M(\mu \mu \ell \ell)$ scattered distribution, as well as the results of the fits to the distributions.

The systematic uncertainties are dominated by the fitting procedures of $4.2 \%$, reconstruction efficiencies of $4.0 \%, Z$ boson background line shape of $3.7 \%$, and $J / \psi$ signal mass shape of $2.8 \%$. The rest of the systematic sources are small. The quadratic sum of systematic uncertainties is calculated to be $7.6 \%$.

The fiducial branching fraction is measured with respect to the $Z \rightarrow 4 \mu$ decay, where part of the systematic uncertainties are cancelled. With a similar selection of $4 \mu$ candidates and a requirement of $4 \mathrm{GeV}<M(\mu \mu)<80 \mathrm{GeV}, 250 \pm 20 Z \rightarrow 4 \mu$ events are obtained as the normalization channel. The ratio of the branching fraction in the fiducial phase-space is given by $\mathscr{R}_{J / \psi \ell^{+} \ell^{-}}=$ $\frac{\mathscr{B}\left(Z \rightarrow J / \psi \ell^{+} \ell^{-}\right)}{\mathscr{B}(Z \rightarrow 4 \mu)}=\left(\frac{1}{2} \sum_{\ell=e, \mu} \frac{N_{Z \rightarrow J / \psi \ell^{+} \ell^{-}}}{\varepsilon_{Z \rightarrow J / \psi \ell^{+} \ell^{-}}}\right) \frac{\varepsilon_{Z \rightarrow 4 \mu}}{N_{Z \rightarrow 4 \mu}} \frac{1}{\mathscr{B}(J / \psi \rightarrow \mu \mu)}=0.67 \pm 0.18 \pm 0.05$, where $\varepsilon_{Z \rightarrow J / \psi \ell^{+} \ell^{-}}$ and $\varepsilon_{Z \rightarrow 4 \mu}$ are the efficiency times acceptance of signal and normalization channel, respectively. The fiducial space are defined by the following requirements: $40<M(\ell \ell)<80 \mathrm{GeV},|\eta(e)|<2.5$, $|\eta(\mu)|<2.4, p_{T}\left(\ell_{1}, \ell_{2}, \mu, \mu\right)>(30,15,3.5,3.5) \mathrm{GeV}$. For signal the $p_{T}$ of $J / \psi$ candidate is required to be above $8.5 \mathrm{GeV}$. If one takes the measured value of $\mathscr{B}(Z \rightarrow 4 \mu)=(1.20 \pm 0.08) \times 10^{-6}$ (with a requirement of $M(\mu \mu)>4 \mathrm{GeV}$ ), the derived branching fraction of $\mathscr{B}\left(Z \rightarrow J / \psi \ell^{+} \ell^{-}\right)$is around $8 \times 10^{-7}$ which agrees with the prediction of SM.
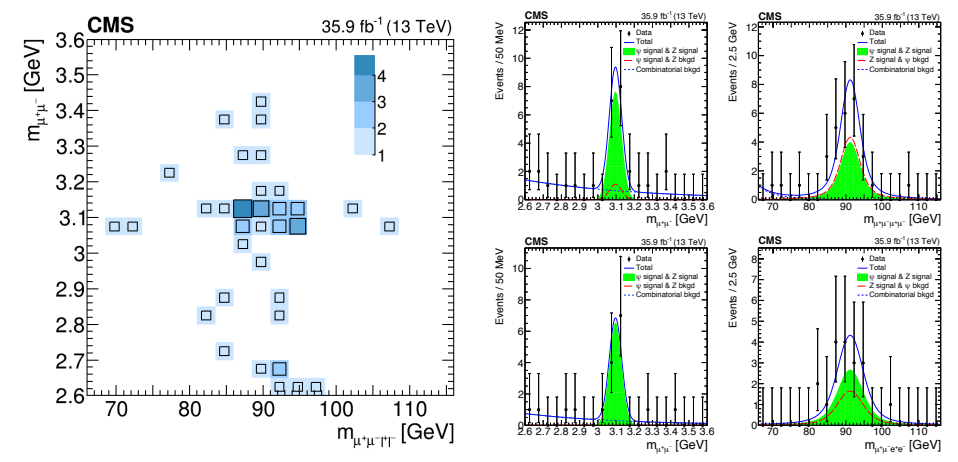

Figure 2: The scattered distribution of $M(\mu \mu)$ and $M(\mu \mu \ell \ell)$ from the selected candidates (left) and the projections to $M(\mu \mu)$ or $M(\mu \mu \ell \ell)$ with the results of the fits superimposed (right), as in Ref. [5].

\section{References}

[1] S. Chatrchyan et al. [CMS Collaboration], JINST 3, S08004 (2008).

[2] C. Bobeth, M. Gorbahn, T. Hermann, M. Misiak, E. Stamou and M. Steinhauser, Phys. Rev. Lett. 112, 101801 (2014) doi:10.1103/PhysRevLett.112.101801 [arXiv:1311.0903 [hep-ph]].

[3] S. Chatrchyan et al. [CMS Collaboration], Phys. Rev. Lett. 111, 101804 (2013) doi:10.1103/PhysRevLett.111.101804 [arXiv:1307.5025 [hep-ex]].

[4] V. Khachatryan et al. [CMS and LHCb Collaborations], Nature 522, 68 (2015) doi:10.1038/nature14474 [arXiv:1411.4413 [hep-ex]].

[5] A. M. Sirunyan et al. [CMS Collaboration], Phys. Rev. Lett. 121, no. 14, 141801 (2018) doi:10.1103/PhysRevLett.121.141801 [arXiv:1806.04213 [hep-ex]]. 